\title{
Can the Mixed Virtual Reality Simulator Into D'mentia Enhance Empathy and Understanding and Decrease Burden in Informal Dementia Caregivers?
}

\author{
Linda Helena Jütten Ruth Elaine Mark Margriet Maria Sitskoorn \\ Department of Cognitive Neuropsychology, Tilburg University, Tilburg, The Netherlands
}

\section{Keywords}

Caregiving · Informal caregiving · Experiential learning · Dementia · Intervention · Simulation training $\cdot$ Technology

\begin{abstract}
Objective: To evaluate whether the mixed virtual reality dementia simulator training Into D'mentia increased informal caregivers' understanding for people with dementia, their empathy, sense of competence, relationship quality with the care receiver, and/or decreased burden, depression, and anxiety. Methods: A quasi-experimental longitudinal study with an intervention group ( $n=145)$ and a control group $(n=56)$ was conducted. All participants were informal caregivers of people with dementia. They completed six questionnaires and semistructured interviews 1 week before as well as 1 week, 2.5 months, and 15 months after the training. Data were analyzed on both group and individual level using linear mixed model analyses and Reliable Change Indices. Results: Eighty-five percent of the participants in the intervention group found the intervention useful; $76 \%$ said they had changed their approach to caregiving, and $61 \%$ stressed that the intervention had increased their understanding of dementia. No significant differences were found between the two groups over time regarding empathy, sense of competence, relationship quality with the care receiver, burden, depression, and anxiety, at either group or individual level. Conclusion: Caregivers indicated that the Into D'mentia intervention improved their understanding of dementia, that they had learned to be more patient, to take things more slowly, and to focus on positive aspects of caregiving. However, no significant change was found on the variables assessed via the questionnaires. Future research can consider enriching this intervention with other aspects such as more educational material, more simulations, and group sessions, tailored to the individual caregiver
\end{abstract}


and his/her situation, and examine whether these new interventions yield change on questionnaires. These new, more personalized interventions for dementia caregivers could help caregivers to better understand the persons with dementia they care for and to ultimately enhance the well-being of both caregivers and persons with dementia.

(C) 2018 The Author(s)

Published by S. Karger AG, Basel

\section{Introduction}

Caregiving can be very burdensome for informal caregivers of people with dementia and may lead to various deleterious consequences, including poorer psychological health [1]. In order to diminish these negative effects, several interventions have been developed [2]. However, few interventions have attempted to increase the caregiver's understanding of and empathy for people with dementia in an attempt to reduce burden, while the benefits of increasing both are thought to be beneficial for professional and informal carers [3-6] and for the person they care for $[6,7]$. The mixed virtual reality Into D'mentia simulator [8], developed in 2010, aims to fill this research gap.

Understanding and knowledge of dementia enable caregivers to deliver high-quality care; they are essential for caregivers to be able to meet the patient's health care needs [6] and can diminish communication problems between the caregiver and the person with dementia [9]. This could in turn reduce caregiver burden and improve relationship quality [10]. Existing interventions have mostly focused on enhancing knowledge and utilized written materials as well as information about coping strategies. Overall, these interventions are only moderately effective in alleviating burden and depression [11]. Caregivers may need more understanding (instead of only knowledge) to accurately appreciate the patients' experience of the problem and in turn be able to meet the patient's health care needs. To reach a higher level of understanding, caregivers might need to look at things from the person with dementia's perspective [6]. This links to cognitive empathy - the ability to understand the other persons' perspective, or theory of mind - and (to a lesser extent) to affective empathy - feeling what another person feels [4]. Cognitive and affective empathy may have different influences on aspects of well-being of informal dementia caregivers. Cognitive empathy has been positively associated with lower stress appraisal, lower threat appraisal, and lower levels of depression among informal caregivers $[5,12]$. Affective empathy has been found to be associated with higher stress appraisal, less life satisfaction, and more anxiety symptoms [5, 12]. These findings suggest that, in addition to enhancing understanding, the intervention's focus should be on enhancing cognitive empathy (and/or reducing affective empathy) to enhance the caregivers' lives.

To date, only two interventions are (partially) aimed at heightening caregivers' empathy: the web-based STAR (Skills Training and Reskilling) e-learning course [13] and the 13-min virtual reality movie of Through the D'mentia Lens (TDL) [14], both of which were moderately effective in heightening (cognitive) empathy. However, both studies used small study samples, and the study about the TDL intervention omitted a control group. While the results are promising, no thorough (longitudinal) study incorporating both an intervention and a control group has attempted to heighten the understanding of and empathy for persons with dementia in order to enhance the caregivers' lives.

In 2010, the mixed virtual reality simulator Into D'mentia was developed, in which dementia caregivers experience what it is like to have dementia [8]. The simulator's goal is to increase the understanding of and empathy for people with dementia in informal caregivers by experiential learning. Experiential learning is learning in which the learner is directly in touch with the realities being studied, instead of only reading, hearing, or writing about it 
[15], and can be achieved by (virtual or augmented) simulation [16]. Simulation training to enhance understanding and empathy has been found to be effective for health care students [17], but has to date not been thoroughly studied in experimental studies with informal caregivers.

The primary aim of the current study was to examine whether the Into D'mentia training increased understanding of dementia and cognitive empathy ( 1 week, 2.5 months, and 15 months after the intervention) in informal caregivers. The secondary aims were to examine whether the Into D'mentia training decreased caregiver burden, depression, and anxiety, and/or enhanced sense of competence and relationship quality with the care receiver. However, the caregiver population is very heterogeneous; caregivers differ from each other on a number of caregiver-related variables (e.g., age, sex, educational level), care receiver variables (e.g., time since diagnosis), and background variables (e.g., time spent on caregiving, whether or not they live with the care receiver). In addition, caregivers differ in their baseline empathy levels. Due to the heterogeneous nature of the group we did not expect the intervention to have the same effects for every caregiver, therefore both group and individual analyses were executed.

\section{Design and Methods}

\section{Study Design}

The current study is a quasi-experimental longitudinal investigation into the effectiveness of the Into D'mentia simulator. The outcome variables were assessed at four time points: 1 week before the Into D'mentia training (T1), 1 week after the training (T2), 2.5 months after the training (T3), and 15 months after the training (T4) to examine both shortand long-term effects. The published protocol of the study [18] describes the method in greater detail.

\section{Participants}

An intervention and a control group, group-matched on sex and level of education, took part. All participants were adult informal caregivers (who spent at least $8 \mathrm{~h}$ per week on caregiving) of a relative, spouse, or friend with dementia who lived at home. The inclusion and exclusion criteria were the same for both groups, while the controls (an attention-only group) did not experience the simulator training. None of the participants or their care receivers were prohibited from usual care.

The recruitment of the intervention group started in July 2014 and ended in August 2014, during which time 192 participants were screened for eligibility and 145 were included. One participant did not complete T2 and 20 participants did not complete T3. At T4, 80 participants dropped out, leaving 65 participants at the final time point. The recruitment of the control group started in May 2015 and ended in January 2017, during which time 75 participants were screened for eligibility and 56 were included. Three participants did not complete T2, and 13 participants did not complete T3. At T4, 34 participants dropped out, leaving 22 participants at the final time point. The participants who did not complete an assessment without giving reasons why were invited to take part in the following measurement(s), so it is possible that a participant did not complete T2, but did complete T3. All available data were analyzed using linear mixed models analyses. Online supplementary Figure S1 (for all online suppl. material, see www.karger.com/doi/10.1159/000494660) shows the flowchart of participants.

Table 1 shows the participant characteristics and pre-intervention scores on the outcome variables. Mean age was 59.6 ( $\mathrm{SD}=11.9$ ) years in the intervention group and $63.8(\mathrm{SD}=11.8)$ 
Table 1. Participant characteristics

\begin{tabular}{|c|c|c|c|c|}
\hline & $\begin{array}{l}\text { Intervention group } \\
(n=145)\end{array}$ & $\begin{array}{l}\text { Control group } \\
(n=56)\end{array}$ & Test value ${ }^{a}$ & $p$ value \\
\hline \multicolumn{5}{|c|}{ Characteristics of the informal caregivers } \\
\hline Age, years & $59.6 \pm 11.9$ & $63.8 \pm 11.8$ & 2.250 & $0.026^{*}$ \\
\hline Male sex & $30(20.7 \%)$ & $13(23.2 \%)$ & 0.153 & 0.704 \\
\hline \multicolumn{5}{|l|}{ Level of education ${ }^{\mathrm{b}}$} \\
\hline Low & $23(15.9 \%)$ & $7(12.5 \%)$ & 0.407 & 0.816 \\
\hline Medium & $52(35.9 \%)$ & $20(35.7 \%)$ & & \\
\hline High & $70(48.2 \%)$ & $29(51.8 \%)$ & & \\
\hline Time providing care a week, $\mathrm{h}$ & $47.2 \pm 60.1$ & $78.6 \pm 65.4$ & -3.232 & $0.001^{* *}$ \\
\hline \multicolumn{5}{|l|}{ Relationship with the care receiver } \\
\hline Spouse & $52(36.1 \%)$ & $30(53.6 \%)$ & 7.415 & $0.025^{*}$ \\
\hline Child & $67(46.5 \%)$ & $23(41.1 \%)$ & & \\
\hline Other & $25(17.4 \%)$ & $3(5.3 \%)$ & & \\
\hline Cohabiting with the care receiver & $46(32.2 \%)$ & $32(57.1 \%)$ & 10.532 & $0.002^{* *}$ \\
\hline \multicolumn{5}{|c|}{ Characteristics of the persons with dementia } \\
\hline Age, years & $78.8 \pm 8.4$ & $78.1 \pm 8.0$ & -0.551 & 0.583 \\
\hline Male sex & $61(42.7 \%)$ & $26(47.3 \%)$ & 0.344 & 0.632 \\
\hline \multicolumn{5}{|l|}{ Dementia diagnosis } \\
\hline Alzheimer's dementia & $86(59.3 \%)$ & $30(58.8 \%)$ & 2.150 & 0.708 \\
\hline Vascular dementia & $23(15.9 \%)$ & $12(23.5 \%)$ & & \\
\hline $\mathrm{PD}$ & $3(2.1 \%)$ & $1(2.0 \%)$ & & \\
\hline Other & $8(5.5 \%)$ & $2(3.9 \%)$ & & \\
\hline No differential diagnosis & $25(17.2 \%)$ & $6(11.8 \%)$ & & \\
\hline Time since diagnosis, years & $3.6 \pm 2.6$ & $2.6 \pm 2.2$ & 1.907 & 0.058 \\
\hline \multicolumn{5}{|c|}{ Pre-intervention scores on the questionnaires } \\
\hline IRI - Perspective Taking ${ }^{c}$ & $17.7 \pm 4.0$ & $17.4 \pm 4.4$ & 0.498 & 0.619 \\
\hline IRI - Empathic Concern ${ }^{c}$ & $17.8 \pm 4.3$ & $17.8 \pm 3.9$ & -0.026 & 0.980 \\
\hline HADS-D ${ }^{\mathrm{d}}$ & $4.8 \pm 4.2$ & $5.5 \pm 3.5$ & -1.113 & 0.268 \\
\hline HADS-A ${ }^{\mathrm{d}}$ & $6.2 \pm 3.8$ & $7.8 \pm 4.3$ & -2.672 & $0.008^{* *}$ \\
\hline CRA - Disruption ${ }^{\mathrm{e}}$ & $2.9 \pm 0.9$ & $3.5 \pm 0.8$ & -4.303 & $<0.001^{* *}$ \\
\hline CRA - Health & $2.4 \pm 0.8$ & $3.0 \pm 0.9$ & -4.609 & $<0.001^{* *}$ \\
\hline CRA - Support ${ }^{\mathrm{e}}$ & $2.3 \pm 0.6$ & $2.7 \pm 0.8$ & -3.358 & $0.001^{* *}$ \\
\hline CRA - Self-Esteem ${ }^{\mathrm{e}}$ & $3.8 \pm 0.6$ & $3.7 \pm 0.6$ & 1.139 & 0.256 \\
\hline CRA - Finances ${ }^{\mathrm{e}}$ & $2.3 \pm 0.6$ & $2.6 \pm 0.8$ & -2.493 & $0.014^{*}$ \\
\hline $\mathrm{RQI}^{\mathrm{f}}$ & $31.6 \pm 7.9$ & $29.4 \pm 8.7$ & 1.637 & 0.103 \\
\hline QoR - Current ${ }^{g}$ & $13.2 \pm 2.9$ & $13.4 \pm 3.0$ & -0.393 & 0.695 \\
\hline $\mathrm{SSCQ}^{\mathrm{h}}$ & $27.3 \pm 5.0$ & $24.1 \pm 5.5$ & 3.851 & $<0.001^{* *}$ \\
\hline
\end{tabular}

Values are presented as mean \pm SD or $n(\%)$. CRA, Caregiver Reaction Assessment; HADS, Hospital Anxiety and Depression Scale; IRI, Interpersonal Reactivity Index; PD, dementia associated with Parkinson's disease; QoR, Quality of Relationship; RQI, Relationship Quality Index; SSCQ, Short Sense of Competence Questionnaire. ${ }^{\text {a }}$ Test values for continuous outcomes: $t$ values for normally distributed variables and standardized statistics for Mann-Whitney U-tests for non-normally distributed variables; for categorical values: Pearson $\chi^{2}$ values. ${ }^{b}$ Educational level according to Verhage [35], recoded as low (1-4), medium (5), or high (6-7). ${ }^{\mathrm{c}}$ Score range $0-28 .{ }^{\mathrm{d}}$ Score range $0-21 .{ }^{\mathrm{e}}$ Score range $1-5 .{ }^{\mathrm{f}}$ Score range $7-42 .{ }^{\mathrm{g}}$ Score range $4-20 .{ }^{\mathrm{h}}$ Score range $7-35$. $* p<0.05,{ }^{* *} p<0.01$

years in the control group, which is a small but significant difference $(p=0.026)$. Most participants were female $79.3 \%$ in the experimental group and $77.8 \%$ in the control group), and most were highly educated $(48.3 \%$ in the experimental group and $51.8 \%$ in the control group). The groups did not differ on these latter two sociodemographic variables. However, participants in the control group spent significantly more time a week on providing care $(p=$ 
$0.001)$, more of them lived with their care receiver $(p=0.002)$, and the control group consisted of a higher proportion of spouses than the intervention group $(p=0.025)$.

There were no differences on the sociodemographic variables between those who completed T3 and those who dropped out before T3 in either group. Regarding the outcome variables, participants of the intervention group who dropped out had significantly higher anxiety scores at $\mathrm{T} 1$ than those who completed $\mathrm{T} 3$ (mean $=8.3, \mathrm{SD}=3.8$ versus mean $=5.8$, $\mathrm{SD}=3.7, p=0.004$ ), and they had better perceived social support (Caregiver Reaction Assessment - Support ( (mean $=1.9, \mathrm{SD}=0.9$ versus mean $=2.3, \mathrm{SD}=0.8 p=0.035$ ). Regarding the control group, the participants who completed T3 had significantly fewer health problems (Caregiver Reaction Assessment - Health) than those who dropped out before $\mathrm{T} 3$ (mean = $2.8, \mathrm{SD}=0.9$ versus mean $=3.6, \mathrm{SD}=0.8, p=0.024$ ). No other significant differences between those who did and did not complete T3 were found. Online supplementary Table S1 shows all comparisons.

There were no differences on the sociodemographic or outcome variables between the participants who completed T4 and those who dropped out before T4 in the intervention group. Regarding the control group, the participants who completed T4 had significantly fewer financial problems at T1 than those who dropped out before T4 (mean $=2.4, \mathrm{SD}=0.9$ versus mean $=2.9, \mathrm{SD}=0.7, p=0.039$ ). No other differences were found. Online supplementary Table $\mathrm{S} 2$ shows all comparisons.

\section{Intervention}

The intervention consisted of a mixed virtual reality dementia simulator training, an individual conversation with the trainer immediately after the simulation, and a group meeting with 8-10 other participants 1-2 weeks later. In the simulator, the participants experienced what it is like to have dementia, as if they walked in the patients' shoes. During the group meeting, the experiences in the simulator were shared and put in perspective. In addition, practical tips and tricks were shared, and the caregivers could learn from each other's caregiving experiences. The control group was an "attention only" group and received no intervention. The protocol describes the intervention in greater detail [18].

\section{Outcome Measures}

Semi-Structured Interview. During the semi-structured interviews at T3 and T4, several questions were asked (to the intervention group only) about the overall experienced usefulness of the intervention, whether the caregivers' understanding of the person with dementia had changed, and whether the caregivers had learned anything from the intervention (Table 2).

Questionnaires - Empathy. Two subscales of the Interpersonal Reactivity Index (IRI) [19] were used to measure empathy: Perspective Taking and Empathic Concern. Perspective Taking measures cognitive empathy, the tendency to take the psychological point of view of others, akin to the "theory of mind" concept (Cronbach's $\alpha=0.670$ ). Empathic Concern measures affective empathy, the ability to feel for others (Cronbach's $\alpha=0.655$ ). Both subscales consist of seven items, each rated on a five-point Likert scale (0-4), with a maximum score of 28 per subscale [20].

Questionnaires - Secondary Outcome Measures. The other outcomes were caregiver burden, measured using the Caregiver Reaction Assessment [21], subscales Impact of Caregiving on Disrupted Schedule, Health Problems, Lack of Family Support, Self-Esteem, and Financial Problems; depression and anxiety, both measured using the Hospital Anxiety and Depression Scale [22]; relationship quality, measured with both the Relationship Quality Index [23] and the Quality of Relationship - Current [24]; and sense of competence, measured with the Short Sense of Competence Questionnaire [25]. 
Table 2. Results of the interview (at T3 and T4)

$\mathrm{T} 3, \% \quad \mathrm{~T} 4, \%$

\section{Simulator}

Does the simulator give an accurate reflection of what a person with dementia goes through?

yes

a bit

no

Did the simulator meet your expectations?

Do you think the simulator is useful?

\begin{tabular}{lrr} 
yes & 46.8 & 73.0 \\
a bit & 18.8 & 20.7 \\
no & 15.6 & 6.3 \\
I had no expectations & 18.8 & \\
\hline yes & 85.2 & 87.1 \\
a bit & 8.6 & 8.1 \\
no & 6.2 & 4.8 \\
\hline
\end{tabular}

\section{Group meeting with other participants after the simulation}

\begin{tabular}{llrr}
\hline Did you feel supported by the experiences and stories of & yes & 79.0 & 62.5 \\
the other participants in the group meeting? & a bit & 14.0 & 29.7 \\
& no & 7.0 & 7.8 \\
\hline Did the group meeting meet your expectations? & yes & 61.2 & 58.0 \\
& a bit & 24.0 & 38.7 \\
& no & 11.6 & 3.3 \\
\hline Do you think the group meeting is useful? & I had no expectations & 3.2 & \\
\hline & yes & 82.2 & 73.4 \\
& a bit & 11.6 & 23.4 \\
\hline
\end{tabular}

Whole training (simulator and group meeting combined)

\begin{tabular}{llrr}
\hline Did the whole training (simulator and group meeting & yes & 70.5 & 78.1 \\
together) have a personal impact on you? & no & 29.5 & 21.9 \\
\hline Do you think that the whole training helps you to be a & yes & 65.9 & 46.8 \\
more effective caregiver? & a bit & 9.3 & 43.8 \\
& no & 24.8 & 9.4 \\
\hline Do you think the whole training has helped you to & yes & 60.5 & 54.7 \\
understand your spouse/relative/friend? & a bit & 14.7 & 37.5 \\
& no & 24.8 & 7.8 \\
\hline Do you think that you are better prepared for what is & yes & 37.2 & 35.9 \\
going to happen in the future? & a bit & 18.6 & 46.9 \\
& no & 44.2 & 17.2 \\
\hline Are you surer of your qualities because of the training? & yes & 56.3 & 37.5 \\
& a bit & 15.6 & 45.3 \\
& no & 28.1 & 17.2 \\
\hline Did you learn anything from the training? & yes & 93.0 & 85.9 \\
& no & 7.0 & 14.1 \\
\hline Do you do anything different in caring because of the & yes & 76.0 & 58.7 \\
training? & no & 24.0 & 41.3 \\
\hline
\end{tabular}




\section{Study Procedure}

All participants completed a semi-structured interview and a questionnaire booklet at each measurement point. Trained neuropsychologists, who were not part of the intervention, conducted the (individual) semi-structured interviews with the caregivers. Due to the content of the semi-structured interviews, the interviewers could not be blinded. However, participants filled out the questionnaires themselves at home, minimizing the interviewers' influence. The participants were blinded to the assigned groups; each groups were unaware of the other group's existence. The control group was debriefed after the last measurement and offered an educational group meeting to compensate for their contribution.

\section{Statistical Analyses}

Statistical analyses were performed using SPSS Statistics 22 [26] and R software, nlme package [27]. Differences between the groups on demographic variables and pre-intervention scores were examined using independent-samples $t$ tests and $\chi^{2}$ tests.

Semi-Structured Interview. The interview questions were analyzed using frequencies.

Questionnaires - Group Change. Comparative analyses between the two groups were conducted using linear mixed models analyses. For all outcome measures separate models were built using a step-up strategy [28]. The restricted maximum likelihood estimate (REML) was used to estimate the model parameters, and the Akaike Information Criterion (AIC) and the Bayesian Information Criterion (BIC) were used to estimate model fit. For each model, the random effect was the intercept, and the fixed effects were group (intervention versus control), time, and the time by group interaction. A quadratic effect of time was tested but found to be neither significant nor improving model fit, and therefore not included. No random slopes were added because these did not improve model fit. A first-order autoregressive covariance structure at level 1 and a scaled identity matrix at level 2 (because there was only one random effect) were selected because these provided the best fit. The groups were matched on sex and level of education, but differed significantly on the variables age, cohabiting with the care receiver, hours spent on care a week, and type of the relationship with the care receiver. These variables (accompanied by their interaction with time) were added consecutively to the full model (described above) to examine whether these improved the model fit. The model fit was estimated by computing the likelihood ratio statistic (using the log-likelihood based on the maximum likelihood and degrees of freedom) [28]. The final models included the covariates age, cohabiting with the person with dementia, and hours spent on care, since these models provided the best fit.

Questionnaires - Individual Change. The Reliable Change Index (RCI) was computed as an indication for clinically significant change [29], from both T1 to T2, T1 to T3, and T1 to T4. The RCI was based on a $90 \% \mathrm{CI}$, which corresponds to a cut-off value of 1.65. $p$ values $<0.05$ were considered statistically significant. However, to adjust for multiple significance testing, the Benjamini-Hochberg procedure (false discovery rate) [30] was applied in case of significant results. This procedure is less strict than the Bonferroni correction and recommended for health-related studies [31].

\section{Results}

\section{Semi-Structured Interview}

Table 2 shows the answers and the questions asked during the semi-structured interviews with the caregivers in the intervention group at $\mathrm{T} 3$ and $\mathrm{T} 4$. At T3, most caregivers found the simulation and group meeting useful ( 85.2 and $82.2 \%$, respectively); $65.9 \%$ thought that the training (simulator and group meeting combined) helped them to be a better caregiver; 
$60.5 \%$ thought the training had helped them to better understand their care receiver; and $56.3 \%$ were surer of their qualities after the training. Ninety-three percent of the caregivers said they had learned something from the training. They indicated that they now had a better understanding of dementia and that they were more aware of the fact that they needed to be more patient with the care receiver. Seventy-six percent indicated that they did something different in caring for their loved one. The caregivers most often mentioned that after the intervention, they took things more slowly, that they had lowered their expectations of the care receiver, that they were learning to let go of disagreements rather than starting or continuing arguments, and that they focused more on the positive side of the caregiving experience and on the things that went well instead of focusing only on the losses.

\section{Questionnaires - Group Change}

Table 3 shows the means and SDs for all questionnaires on each time point and the results of the linear mixed models analyses. No significant differences were found between the groups over time for either primary outcome variable: Perspective Taking, $\beta=0.218, p=$ 0.381 ; Empathic Concern, $\beta=0.047, p=0.862$. For the secondary outcome variables, no significant results were found either.

\section{Questionnaires - Individual Change}

Tables 4 and 5 as well as online supplementary Table S3 show the RCIs for both groups and all outcome measures for T1-T2, T1-T3, and T1-T4, respectively. Approximately the same number of participants show "reliable change" (both "positively" and "negatively") on all outcome measures on all time intervals for both groups.

\section{Discussion}

The current study assessed whether the Into D'mentia simulator training could heighten empathy for, and understanding of, people with dementia among informal caregivers at both group and individual level. In addition, it examined whether the intervention could decrease symptoms of depression, anxiety, and burden, and/or enhance sense of competence and/or relationship quality between caregiver and care receiver. Most participants who underwent the intervention found it useful, indicated that it had helped to increase their understanding of dementia, and said that it had made them a more effective caregiver. However, no significant differences emerged between the two groups (intervention versus control) on the other outcome measures assessed via questionnaires.

For the caregivers who underwent the training, the understanding of dementia was heightened; almost all caregivers indicated that they had learned something from the training, and most also stated that they had changed the way they cared for the person with dementia. While social desirability response bias has to be taken into account when interpreting these findings, these results are promising because how caregivers experience caregiving is crucial and arguably more important than change on a questionnaire [32]. Heightening caregivers' understanding for people with dementia can be beneficial for both caregivers and people with dementia. For the people with dementia, caregivers' heightened understanding can lead to feelings of being important and to a better connection or relationship with the caregiver. In addition, adequate understanding of the problems of the person with dementia can enable the caregivers in meeting the patient's health care needs [6]. For the caregivers, enhanced understanding can lead to fewer communication problems [9], which in turn can lead to a better relationship and enhanced well-being. In addition, the caregivers claimed that they had changed the way they cared for the person with dementia, that they were taking things more 
广


석

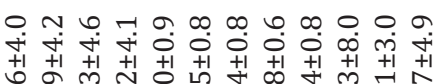
$\stackrel{m}{*}$

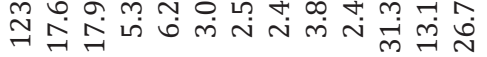



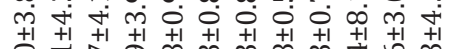

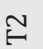

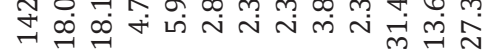

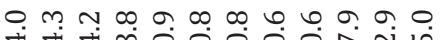

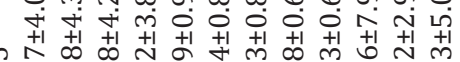

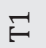

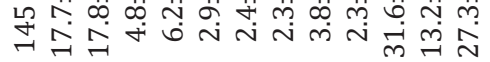

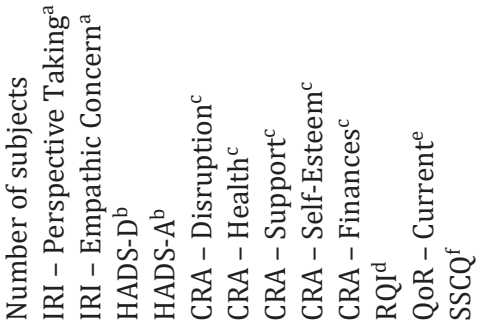

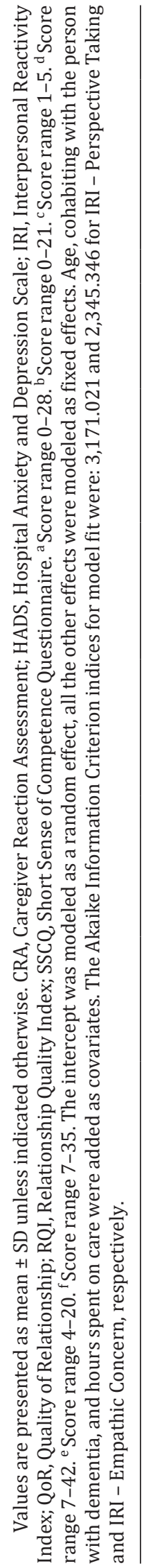


Table 4. RCIs for all outcome measures and both groups, T1-T2

\begin{tabular}{|c|c|c|c|c|}
\hline & \multicolumn{2}{|c|}{ Intervention group } & \multicolumn{2}{|l|}{ Control group } \\
\hline & $\begin{array}{l}\text { reliably changed } \\
\text { (negatively), } n\end{array}$ & $\begin{array}{l}\text { reliably changed } \\
\text { (positively), } n\end{array}$ & $\begin{array}{l}\text { reliably changed } \\
\text { (negatively), } n\end{array}$ & $\begin{array}{l}\text { reliably changed } \\
\text { (positively), } n\end{array}$ \\
\hline IRI - Perspective Taking & 2 (of 141) & 9 (of 141) & 3 (of 51) & $4($ of 51$)$ \\
\hline IRI - Empathic Concern & 7 (of 140) & 5 (of 140) & 1 (of 50) & 2 (of 50) \\
\hline HADS-D & 7 (of 130) & 6 (of 130) & 5 (of 50) & 3 (of 50) \\
\hline HADS-A & 5 (of 134) & 5 (of 134) & 1 (of 50) & $4($ of 50$)$ \\
\hline CRA - Disruption & 4 (of 139) & 5 (of 139) & 3 (of 50) & $2($ of 50$)$ \\
\hline CRA - Health & 5 (of 139) & 5 (of 139) & 3 (of 52) & 4 (of 52) \\
\hline CRA - Support & 7 (of 139) & 2 (of 139) & 1 (of 47) & 4 (of 47) \\
\hline CRA - Self-Esteem & 6 (of 137) & 5 (of 137) & 1 (of 52$)$ & 2 (of 52) \\
\hline CRA - Finances & 12 (of 138) & 16 (of 138) & 6 (of 53) & 6 (of 53) \\
\hline RQI & 8 (of 125) & 4 (of 125) & 2 (of 49 ) & 1 (of 49 ) \\
\hline QoR - Current & 5 (of 110) & 12 (of 110) & 3 (of 44) & 4 (of 44) \\
\hline SSCQ & 5 (of 138$)$ & 3 (of 138) & 2 (of 53) & 3 (of 53) \\
\hline
\end{tabular}

RCIs were based on a $90 \%$ confidence interval. All scores were recoded so that negative change indicates change for the worse, e.g., less empathy, more depression, more burden, and positive change indicates change for the better, e.g., less depression, more empathy, etc. CRA, Caregiver Reaction Assessment; HADS, Hospital Anxiety and Depression Scale; IRI, Interpersonal Reactivity Index; QoR, Quality of Relationship; RCI, Reliable Change Index; RQI, Relationship Quality Index; SSCQ, Short Sense of Competence Questionnaire.

Table 5. RCIs for all outcome measures and both groups, T1-T3

\begin{tabular}{|c|c|c|c|c|}
\hline & \multicolumn{2}{|c|}{ Intervention group } & \multicolumn{2}{|l|}{ Control group } \\
\hline & $\begin{array}{l}\text { reliably changed } \\
\text { (negatively), } n\end{array}$ & $\begin{array}{l}\text { reliably changed } \\
\text { (positively), } n\end{array}$ & $\begin{array}{l}\text { reliably changed } \\
\text { (negatively), } n\end{array}$ & $\begin{array}{l}\text { reliably changed } \\
\text { (positively), } n\end{array}$ \\
\hline IRI - Perspective Taking & 4 (of 123) & 2 (of 123$)$ & 0 (of 40$)$ & 2 (of 4$)$ \\
\hline IRI - Empathic Concern & 4 (of 119) & 6 (of 119) & 1 (of 40$)$ & $2($ of 40$)$ \\
\hline HADS-D & 10 (of 112) & 2 (of 112) & 1 (of 43) & 4 (of 43) \\
\hline HADS-A & 4 (of 117) & 3 (of 117) & 2 (of 43) & 2 (of 43) \\
\hline CRA - Disruption & 8 (of 121) & $6($ of 121$)$ & 5 (of 42) & 0 (of 42$)$ \\
\hline CRA - Health & 8 (of 122) & $4($ of 122$)$ & 4 (of 42) & $2($ of 42$)$ \\
\hline CRA - Support & 11 (of 120 ) & 7 (of 120) & 4 (of 39) & 0 (of 39) \\
\hline CRA - Self-Esteem & $4($ of 120$)$ & $6($ of 120$)$ & 4 (of 41) & $2($ of 41$)$ \\
\hline CRA - Finances & 17 (of 120) & $6($ of 120$)$ & 4 (of 43) & 3 (of 43$)$ \\
\hline RQI & 4 (of 107) & 5 (of 107) & 1 (of 38) & 2 (of 38) \\
\hline QoR - Current & 4 (of 98) & 4 (of 98) & 3 (of 33) & 1 (of 33) \\
\hline SSCQ & $4($ of 121$)$ & 2 (of 102) & 3 (of 43) & 1 (of 43$)$ \\
\hline
\end{tabular}

RCIs were based on a $90 \%$ confidence interval. All scores were recoded so that negative change indicates change for the worse, e.g., less empathy, more depression, more burden, and positive change indicates change for the better, e.g., less depression, more empathy, etc. CRA, Caregiver Reaction Assessment; HADS, Hospital Anxiety and Depression Scale; IRI, Interpersonal Reactivity Index; QoR, Quality of Relationship; RCI, Reliable Change Index; RQI, Relationship Quality Index; SSCQ, Short Sense of Competence Questionnaire.

slowly, and that they had learned to let go of disagreements rather than starting or continuing arguments. This is also promising because it can lead to a more positive living situation for both the caregiver and the person with dementia.

We did not find significant change on the included questionnaires. The two previous intervention studies focused on increasing empathy (measured with the IRI) in dementia 
caregivers - the web-based skills training (STAR) [13] on both cognitive and affective empathy and TDL [14] on cognitive empathy - found significant increases at group level. However, these interventions are not directly comparable to ours: the intervention group of the STAR training consisted of both informal caregivers and volunteers in dementia care, had a small sample size (intervention group: $n=27$; control group: $n=32$ ), and used a web-based portal, consisting of skill-building modules as well as peer and expert groups for support and information exchange, and no simulation techniques to heighten empathy. The TDL $(n=35)$ did use simulation techniques (using virtual reality glasses) and an e-learning course, but did not include a control group. The change in cognitive empathy in that study could therefore be due to other factors (e.g., the passing of time) rather than to the intervention per se.

A systematic review [17] suggested that simulation may be an appropriate educational methodology for developing understanding, empathy, and/or empathic behaviors in health care students. However, there was a large variety in the effect sizes among the reviewed studies. The interventions showing larger effects included a range of educational features, such as role playing, and accompanying feedback. The Into D'mentia intervention did include a group session in which best practices were shared, but role playing and feedback were not included. Maybe if the Into D'mentia intervention were extended by including more educational aspects, changes on the questionnaires would emerge.

The absence of change on the questionnaires between the groups over time in our study could be due to a number of other issues. First, the intervention consisted of one short (20 min) simulation experience and one (half day) group meeting. Several caregivers noted during the interviews that they would have liked more group meetings or a booster session, including more support, and practical tips and tricks to help them in their caregiving tasks. Second, the simulation focuses on the beginning stage(s) of (primarily Alzheimer's) dementia. However, the majority of the caregivers in the intervention group had been a caregiver for more than a year, and several caregivers noted that the simulation was not applicable to their situation anymore because the dementia of their loved one had progressed and other dementia-associated problems had arisen. It is possible that changes on the questionnaires would emerge if the Into D'mentia intervention included more simulation and group sessions specifically designed for specific types of dementia (e.g., Alzheimer's dementia, vascular dementia) and the different stages (mild, moderate, severe) as the dementia progresses, more educational elements, and more specific tips and tricks that caregivers could use in their dayto-day care for their loved one with dementia. Third, in the Netherlands, there are a number of supportive services available for caregivers which are viewed as "usual care." As a consequence, the control participants in our study also had support, which may have resulted in a limited contrast between the two groups. However, it would have been unethical and unfeasible to deprive our participants of usual care.

On the other hand, the lack of change on the questionnaires could be due to methodological issues. First, due to practical issues, the participants were not randomized [18]. In order to overcome this, the groups were (successfully) group-matched on sex and level of education. However, the control group was slightly (but significantly) older than the intervention group, spent more hours on caregiving, and experienced more anxiety and burden than the intervention group. In addition, the control group was considerably smaller than the intervention group, leading to lower statistical power. This was due to difficulties in recruiting control participants; at the time the recruitment started, many caregivers in our recruitment region were already taking part in scientific studies including informal caregivers and were as such not eligible for (or willing to participate in) our control group. Also, there was a large dropout rate, leading to even smaller sample sizes at the last measurement. In addition, while the caregivers who dropped out and completed all measurements did not differ on most of the measured variables, they could be different in unobserved characteristics. Another 
possible explanation lies in the questionnaires used. To measure empathy, we used the IRI, arguably the most frequently used measure to assess empathy, especially in the general (not professional/medical) population. However, despite its wide usage, some of our caregivers found the questions difficult to answer, even after further clarification of specific questions that was given to them if asked for during the interviews. In addition, some researchers argue that the IRI measures trait empathy $[33,34]$ rather than state empathy, and in hindsight this questionnaire might not have been the best measure to assess change. Moreover, since (empathy) questionnaires rely on self-report, one could argue that only the appraisal of empathy was measured - the caregivers' opinion about how they would feel or act in a certain situation rather than the emotion or behavior itself. A means to overcome these questionnaire-related problems is to also include objective measures of empathy to complement the IRI. This way, it would also be possible to assess whether these measure the same construct or not. No objective empathy measures were used in this study because filling out the questionnaires already was time consuming for the participants and we were trying to keep the time investment to a minimum. The original idea of the current study was to also develop a prediction model for change [18]. However, due to the absence of change on the questionnaires, this idea was abandoned.

Despite these limitations, this study has direct relevance for both (clinical) practice and research. The intervention contributed to enhancing caregivers' understanding of (people with) dementia and helped them to feel more effective in caring for their loved one. If the clinician's goal is to heighten understanding and improve the caregivers' experience, this intervention is useful. Future research can consider enriching this intervention with other aspects such as educational material, more simulations, and (group) sessions, tailored to the individual caregiver and his/her situation, and examine whether these new interventions also yield change on questionnaires. These new, more personalized interventions designed for dementia caregivers could go a long way in helping caregivers to better understand the persons with dementia they care for. This could in turn not only assist the caregivers in their caregiving tasks, but also enhance their own well-being and that of the person with dementia they care for.

\section{Acknowledgments}

The authors thank the Department of Psychiatry, VU University Medical Center, Amsterdam Department of Psychiatry; De Wever Tilburg; IJsfontein, Amsterdam; Ideon, Amersfoort; and Stichting Into D'mentia for their help in developing the Into D'mentia simulator training. They would especially like to thank all the informal caregivers who participated in this study for their time and openness regarding their caregiving experiences.

\section{Statement of Ethics}

Caregivers' written informed consent was obtained.

\section{Disclosure Statement}

The authors have no conflict of interest to declare. 


\section{Funding Sources}

This work was supported by the program Memorabel of The Netherlands Organization for Health Research and Development (ZonMw), part of The Netherlands Organization for Scientific Research (NWO) and Alzheimer Nederland. However, solely the authors are responsible for the design and conduct of this study, all analyses, as well as the drafting and editing of this article.

\section{Author Contributions}

L.H. Jütten, R.E. Mark, and M.M. Sitskoorn contributed to study concept and design, participant and data acquisition, and data interpretation. L.H. Jütten executed the statistical analyses and wrote the first draft of the manuscript. The other authors provided critical feedback during the development of the manuscript and approved the final draft. All authors agree to be accountable for all aspects of the work and ensure that questions related to the accuracy or integrity of any part of the work were appropriately investigated and resolved.

\section{References}

1 Pinquart M, Sörensen S. Correlates of physical health of informal caregivers: a meta-analysis. J Gerontol B Psychol Sci Soc Sci. 2007 Mar;62(2):126-37.

2 Gilhooly KJ, Gilhooly ML, Sullivan MP, McIntyre A, Wilson L, Harding E, et al. A meta-review of stress, coping and interventions in dementia and dementia caregiving. BMC Geriatr. 2016 May;16(1):106.

3 Baron-Cohen S, Wheelwright S. The empathy quotient: an investigation of adults with Asperger syndrome or high functioning autism, and normal sex differences. J Autism Dev Disord. 2004 Apr;34(2):163-75.

4 Decety J, Jackson PL. The functional architecture of human empathy. Behav Cogn Neurosci Rev. 2004 Jun;3(2): 71-100.

5 Jütten LH, Mark RE, Sitskoorn MM. Empathy in informal dementia caregivers and its relationship with depression, anxiety, and burden. Int J Clin Health Psychol. Forthcoming 2018.

6 Shattell MM, McAllister S, Hogan B, Thomas SP. "She took the time to make sure she understood": mental health patients' experiences of being understood. Arch Psychiatr Nurs. 2006 Oct;20(5):234-41.

7 Derksen F, Bensing J, Lagro-Janssen A. Effectiveness of empathy in general practice: a systematic review. Br J Gen Pract. 2013 Jan;63(606):e76-84.

8 Hattink BJ, Meiland FJ, Campman CA, Rietsema J, Sitskoorn M, Dröes RM. Experiencing dementia: evaluation of Into D'mentia [article in Dutch]. Tijdschr Gerontol Geriatr. 2015 Oct;46(5):262-81.

9 Dunkin JJ, Anderson-Hanley C. Dementia caregiver burden: a review of the literature and guidelines for assessment and intervention. Neurology. 1998 Jul;51(1 Suppl 1):S53-60.

10 Savundranayagam MY, Hummert ML, Montgomery RJ. Investigating the effects of communication problems on caregiver burden. J Gerontol B Psychol Sci Soc Sci. 2005 Jan;60(1):S48-55.

11 Jensen M, Agbata IN, Canavan M, McCarthy G. Effectiveness of educational interventions for informal caregivers of individuals with dementia residing in the community: systematic review and meta-analysis of randomised controlled trials. Int J Geriatr Psychiatry. 2015 Feb;30(2):130-43.

12 Lee HS, Brennan PF, Daly BJ. Relationship of empathy to appraisal, depression, life satisfaction, and physical health in informal caregivers of older adults. Res Nurs Health. 2001 Feb;24(1):44-56.

13 Hattink B, Meiland F, van der Roest H, Kevern P, Abiuso F, Bengtsson J, et al. Web-based STAR e-learning course increases empathy and understanding in dementia caregivers: results from a randomized controlled trial in the Netherlands and the United Kingdom. J Med Internet Res. 2015 Oct;17(10):e241.

14 Wijma EM, Veerbeek MA, Prins M, Pot AM, Willemse BM. A virtual reality intervention to improve the understanding and empathy for people with dementia in informal caregivers: results of a pilot study. Aging Ment Health. 2018 Sep;22(9):1115-23.

15 Keeton M, Tate P. Learning by experience: What, why, how. New directions for experiential learning, No. 1. San Francisco (CA): Jossey-Bass, Inc; 1978.

16 Medley CF, Horne C. Using simulation technology for undergraduate nursing education. J Nurs Educ. 2005 Jan; 44(1):31-4.

17 Bearman M, Palermo C, Allen LM, Williams B. Learning empathy through simulation: a systematic literature review. Simul Healthc. 2015 Oct;10(5):308-19. 
18 Jütten LH, Mark RE, Maria Janssen BWJ, Rietsema J, Dröes RM, Sitskoorn MM. Testing the effectivity of the mixed virtual reality training Into D'mentia for informal caregivers of people with dementia: protocol for a longitudinal, quasi-experimental study. BMJ Open. 2017 Aug; 7(8):e015702.

19 Davis MH. A multidimensional approach to individual differences in empathy. J South Afr Stud. 1980;10: 85-104.

20 Davis MH. Measuring individual differences in empathy: evidence for a multidimensional approach. J Pers Soc Psychol. 1983;44(1):113-26.

21 Nijboer C, Triemstra M, Tempelaar R, Sanderman R, van den Bos GA. Measuring both negative and positive reactions to giving care to cancer patients: psychometric qualities of the Caregiver Reaction Assessment (CRA). Soc Sci Med. 1999 May;48(9):1259-69.

22 Spinhoven P, Ormel J, Sloekers PP, Kempen GI, Speckens AE, Van Hemert AM. A validation study of the Hospital Anxiety and Depression Scale (HADS) in different groups of Dutch subjects. Psychol Med. 1997 Mar;27(2): 363-70.

23 Norton R. Measuring marital quality: A critical look at the dependent variable. J Marriage Fam. 1983;45(1): 141-51.

24 Bengtson VL. Longitudinal Study of Generations, 1971, 1985, 1988, 1991, 1994, 1997: [California] (ICPSR 4076). Ann Arbor, MI: Inter-university Consortium for Political and Social Research; 2009.

25 Vernooij-Dassen MJ, Felling AJ, Brummelkamp E, Dauzenberg MG, van den Bos GA, Grol R. Assessment of caregiver's competence in dealing with the burden of caregiving for a dementia patient: a Short Sense of Competence Questionnaire (SSCQ) suitable for clinical practice. J Am Geriatr Soc. 1999 Feb;47(2):256-7.

26 SPSS Inc. IBM SPSS Statistics for Windows, Version 22.0. Armonk (NY): IBM Corp.; 2013.

27 Pinheiro J, Bates D, DebRoy S, Sarkar D; R Core Team. nlme: Linear and Nonlinear Mixed Effects Models. R package version 3.1-137. 2018. https://CRAN.R-project.org/package=nlme.

28 West BT, Galecki AT, Welch KB. Linear mixed models. New York: CRC Press; 2014.

29 Jacobson NS, Truax P. Clinical significance: a statistical approach to defining meaningful change in psychotherapy research. J Consult Clin Psychol. 1991 Feb;59(1):12-9.

30 Benjamini Y, Hochberg Y. Controlling the false discovery rate: a practical and powerful approach to multiple testing. J R Stat Soc Series B Stat Methodol. 1995 57:289-300.

31 Glickman ME, Rao SR, Schultz MR. False discovery rate control is a recommended alternative to Bonferronitype adjustments in health studies. J Clin Epidemiol. 2014 Aug;67(8):850-7.

32 Sandelowski M. Focus on qualitative methods: using qualitative methods in intervention studies. Res Nurs Health. 1996 Aug;19(4):359-64.

33 Koller I, Lamm C. Item response model investigation of the (German) interpersonal reactivity index empathy questionnaire. Eur J Psychol Assess. 2015 31:211-21.

34 Devlin HC, Zaki J, Ong DC, Gruber J. Not as good as you think? Trait positive emotion is associated with increased self-reported empathy but decreased empathic performance. PLoS One. 2014 Oct;9(10):e110470.

35 Verhage F. Intelligentie en leeftijd: Onderzoek bij Nederlanders van twaalf tot zevenenzeventig jaar. Assen: Van Gorcum; 1964. 